\title{
Performance of Semi-active/Passive Integrated Isolator based on Magnetorheological Elastomer and Spring
}

\author{
Guanglei Du${ }^{1}$, Xuegong Huang ${ }^{1}$ a), Yancheng $\mathrm{Li}^{2}$, Qing Ouyang ${ }^{1}$, Jiong Wang ${ }^{1}$ \\ ${ }^{1}$ School of Mechanical Engineering, Nanjing University of Science and Technology, Nanjing 210094, China \\ ${ }^{2}$ School of Civil and Environmental Engineering, University of Technology Sydney, Ultimo 2007, Australia \\ E-mail: huangxg@njust.edu.cn
}

\begin{abstract}
The paper reports an investigation on a semi-active/passive integrated vibration isolator utilizing magnetorheological elastomer (MRE) and a spring. To overcome the main shortcoming of passive isolation system, i.e. lack of adaptability, the semi-active/passive integrated isolator (SAPII) based on MRE and spring is designed and prototyped. The magnetic circuit is optimized by finite element analysis to fully unlock the unique feature of MRE. The dynamic response characteristic of the SAPII is experimentally investigated under sweep frequency test. A dynamic model of the SAPII vibration isolation system is established on the basis of Kelvin model. The model parameters, such as equivalent stiffness and equivalent damping, are identified from experimental data. A ON-OFF control law based on the minimal displacement transmissibility is designed for isolation control of the sinusoid excitation. Two control laws, i.e. ON-OFF control and fuzzy logic control, are designed for vibration isolation of random excitation. Finally, the effectiveness of these control laws is verified by numerical simulation and experiment.
\end{abstract}

Keywords: magnetorheological elastomer (MRE), vibration isolation, semi-active, passive, transmissibility control, fuzzy control

\section{1 .Introduction}

Passive vibration isolation is a simple and reliable way of suppressing the vibrations in a dynamic system [1], which is the recommended approach to mitigate unwanted disturbances [2]. The passive isolation systems normally utilize a variety of implementation devices containing viscoelastic materials, springs, hydraulic dampers and pneumatic isolator [3]. However, the fixed natural frequency of passive isolators limits its implementation over wide frequency range. The limitations of passive isolators can be overcome by active or semi-active isolation methods [4]. The bulky and complex nature of the active system makes semi-active isolation as a viable option [5]. In recent years, smart materials have gained much attention for their potential in designing more compact and simpler semi-active isolation systems [6].

Magnetorheological elastomer (MRE), belonging to magnetorheological material family, is a composite material with magnetic-sensitive particles suspended or arranged within non-magnetic elastomer matrix [7]. The comprising rubbery polymers loaded with magnetizable particles that can be aligned along a magnetic field, possess dynamic stiffness and damping that can subsequently be controlled by applied magnetic fields [8]. As a result, vibration isolators containing

a) Author to whom any correspondence should be addressed. 
MRE materials could vary its stiffness in real time under the influence of magnetic field to adapt different frequency of vibrations [9-10]. The unique magnetic field dependent property enhancements make MRE a potential material for vibration isolation which can be achieved either by shifting the resonance region of the structure or by reducing the amplitude of vibration of the structure at resonance [11]. Meanwhile, MRE has very fast response time in the order of milliseconds [8], which can ensure the capacity of real-time control of MRE isolation system.

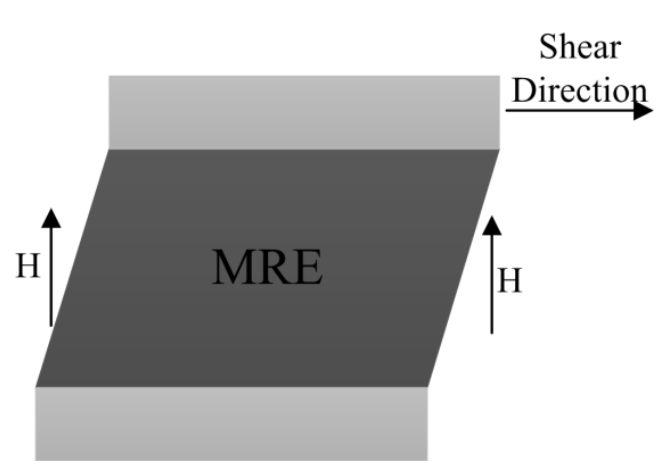

(a)



(b)

Figure 1: The main operation modes of MRE: (a) shear mode, and (b) squeeze/elongation mode.

There are two main operation modes of MRE: shear mode and squeeze/elongation mode, as shown in figure 1. The MRE devices working in shear mode include vibration absorbers [12-15], vibration isolators [16-19] and base isolators [9, 20-21]. Examples of squeeze/elongation mode devices are vibration absorbers [22-23], engineering mounts [24] and compressive spring elements [25]. MREs can operate in a wide range of frequencies and tolerate large shear deformations [11]. Du et al [26] proposed a MRE isolator for controllable automotive seat stiffness of the non-polar conversion system, which demonstrated good isolation effect on random and collision traffic conditions. Kavlicoglu et al [24] implemented a MRE vibration isolation device that can provide a wide range of controllable static stiffness under changing magnetic field. Jeong et al [27] designed a MRE differential mount with variable stiffness to be used in vibration isolation between engine and car body of vehicles. However, most researches are considering MRE as main medium for vibration isolation. As known, on the quest of wider isolation range, the MRE material needs to possess large MR effect. The feasible way to achieve this is to have soft elastomer base therefore possessing big modulus increase. The soft rubber medium, on the other hand, constrains the workability of the MRE isolator when no magnetic field is applied, i.e. passive isolation, due to its low modulus. Most of the isolators can only be used in the applications with small payload.

The greatest advantage of MRE devices is its fast, reversible and controllable stiffness. To fully utilize this feature, efficient and effective control law is the key to realize optimal isolation performance [28]. To this end, Li $\mathrm{W}$ et al [18] proposed an ON-OFF control law for an MRE isolator used in a seat suspension system. Liao G et al [19] developed different ON-OFF control laws for sinusoid excitation, random excitation and impact excitation, whose effectiveness were verified by simulation and experiment. Opie et al [29] developed a clipped-optimal controller for an MRE isolation system with $5-10 \mathrm{~Hz}$ excitation. However, the explorations are still limited and further efforts are required to adventure such journey to pave the way to future implementation.

In this paper, a semi-active/passive integrated isolator (SAPII) utilizing MRE and spring is designed and prototyped to realize the reliable passive isolation and effective semi-active isolation. The organization of the paper is presented as followings: In section 2, the new design of the SPAII is introduced and the magnetic circuit of SAPII is analyzed by the finite element software Maxwell; An experimental setup is developed to obtain the dynamic response characteristic of the SAPII in section 3; In section 4, dynamic model of the vibration isolation system is established, and the parameters of the SAPII are identified based on the dynamic performance and the displacement transmissibility curves under different 
currents. In section 5, the ON-OFF control law based on the minimum principle of displacement transmissibility is designed for isolation control of sinusoid excitation. The ON-OFF control law and the fuzzy logic controller based on damping control and stiffness control are designed for isolation control of random excitation; the effectiveness of these control laws is verified by numerical simulation and experiment shown in section 6 .

\section{2 .The design of the SAPII}

The proposed SAPII consists of one spring and two pieces of MRE used as passive vibration isolation element and semi-active vibration isolation element, respectively, shown in figure 2. Two pieces of MREs have identical dimension of $30 \mathrm{~mm} \times 20 \mathrm{~mm} \times 10 \mathrm{~mm}$. The mass fraction of carbonyl iron particles (Type MPS-MRF-15, Tianyi, China), silicone rubber (Type RTV-704, Kafuter, China) and dimethylsilicone oil (Type D104767, Aladdin, China) are 76\%, 17\% and 7\%, respectively. The MREs are solidified in a magnetic adding device [30], which ensures the anisotropy property. The MREs and the support block form a sandwich structure pre-tensioned by a clamping mechanism.

The sandwich structure ensures MREs working in shear mode when the support block with payload moves up and down. The square-shape magnetic path of the isolator is made of pure iron, type DT4A, which has great magnetic permeability excellent demagnetization property. The platform connected with the support block is used as working plat. A spring is placed underneath the support block to withstand its weight as well as provide passive isolation for the system. Four coils are wound around magnetic path to provide strong magnetic field to MREs. The controlled current range is $0-2 \mathrm{~A}$. Through varying the magnetic field, the stiffness and damping of the MRE can be changed to shift the nature frequency of the SAPII vibration isolation system.

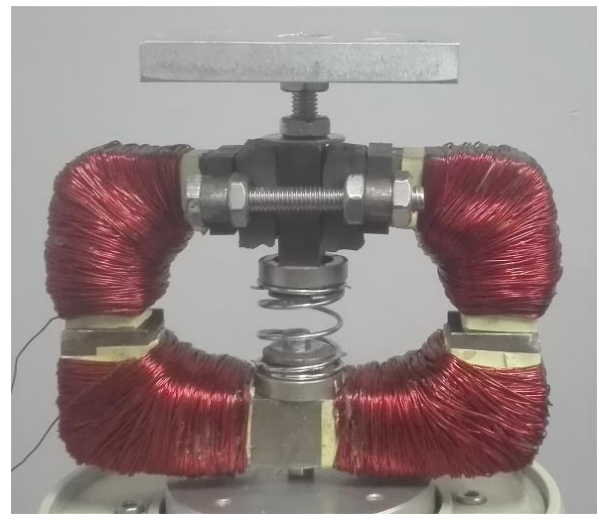

(a)

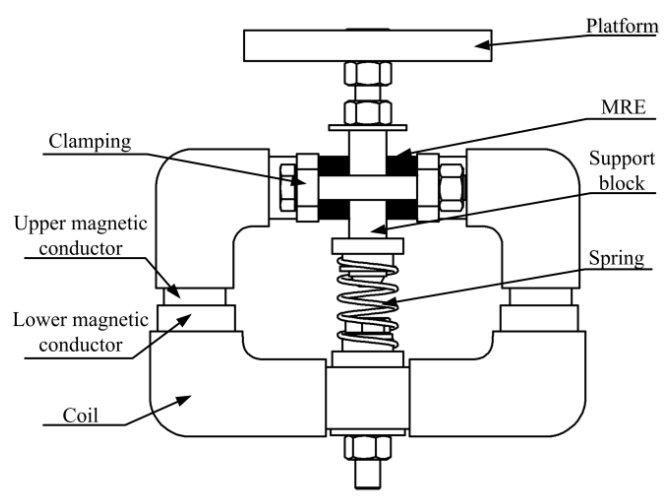

(b)

Figure 2: The structure of the SCII:(a) the prototype and (b) the schematic diagram

This design adopts the most effective and efficient magnetic circuit design, i.e. the $\mathrm{C}$-shape magnetic circuit, since it creates an enclosed field path for the magnetic flux and thus has minimum energy losses [31]. The magnetic flux line and magnetic field intensity in the SAPII are obtained by Ansoft Maxwell 3D shown in figure 3. Under applied current of $2.0 \mathrm{~A}$, the average magnetic field in the magnetic path is $1.875 \mathrm{~T}$, which is lower than the saturation magnetic flux density of DT4A, i.e. 2.5T. The magnetic field in the MRE is perpendicular to the shear direction. The magnetic field in MRE is strong and uniform with average field intensity of $428.5 \mathrm{mT}$ which is very close to the saturation magnetic magnitude of MRE with similar composition [32-33]. 




Figure 3: The magnetic flux line and field intensity in the SAPII $(\mathrm{I}=2.0 \mathrm{~A})$

\section{Experimental testing of the SAPII}

The dynamic response characteristics of the SAPII are studied using a forced vibration test system shown in Figure 4. The signal generated by the signal generator (Model AFG3022, Tektronix, USA) is amplified by the power amplifier (Model YE5872, Sinocera Piezotronics, China) to drive the vibration exciter (Model JZK-10, Sinocera Piezotronics, China). The SAPII is mounted on the exciting head by a screw. The excitation is applied to the isolator via the platform of the SAPII. The acceleration of the loading is measured by an acceleration sensor (Model YD-107, Sinocera Piezotronics, China). A laser displacement sensor (ModelHG-C1100, Panasonic, Japan) is mounted on the reference bar to measure the displacement of platform and excitation. The displacement signal amplified by the charge amplifier (Model 5011, KISTLER, Switzerland) and the acceleration signal are collected by the dynamic test and analysis system (Model SCADAS III, LMS, Belgium). The software-LMS Test .Lab is used to process and analyze the signals. DC power (Model LPS-305, Motech, China) provides constant DC currents to the coils.

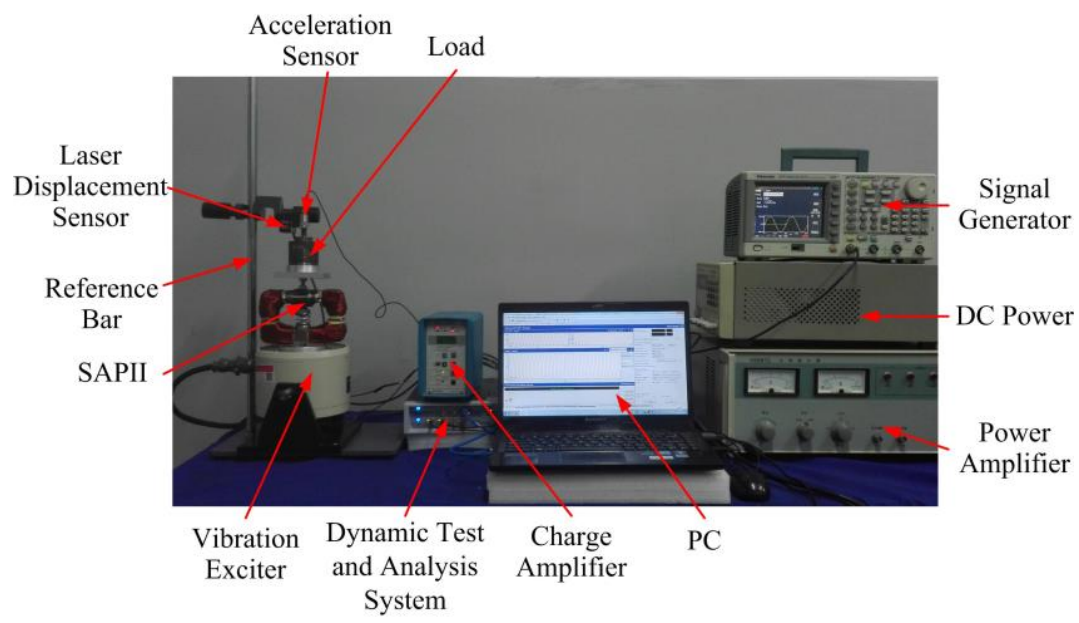

Figure 4: Experimental setup for the SAPII 


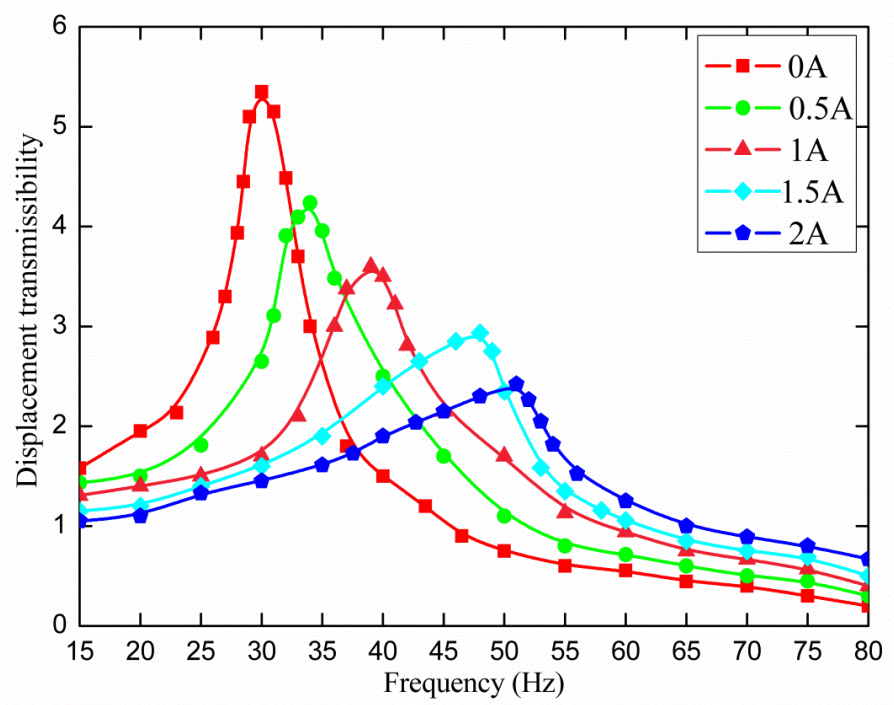

Figure 5:The diplacement transmissibility curves under different currents

During the test, sweep frequency excitation ranging from $15 \mathrm{~Hz}$ to $100 \mathrm{~Hz}$ is applied to the isolator under various applied currents, i.e. $0 \mathrm{~A}, 0.5 \mathrm{~A}, 1.0 \mathrm{~A}, 1.5 \mathrm{~A}$ and $2.0 \mathrm{~A}$. The displacement transmissibility curves under different currents are shown in figure 5. When current is changed from $0 \mathrm{~A}$ to $2 \mathrm{~A}$, the resonant frequencies of the SPAII system shift rather quickly from $30.0 \mathrm{~Hz}$ to $51.0 \mathrm{~Hz}$ with the relative increase of $70 \%$. The peak displacement transmissibility ratios decrease from 5.348 to 2.421 , and the relative change is about $54.7 \%$. The transmissibility curves between $1.5 \mathrm{~A}$ and $2 \mathrm{~A}$ are very close, which indicates the magnetic saturation of the MR elastomer would occur around this range. Figures 6 (a)-(e) illustrate the acceleration amplitude spectrum of the SAPII isolator under various applied current under sweep-frequency excitations eanging from $15 \mathrm{~Hz}$ to $100 \mathrm{~Hz}$. Figure 6 (f) sumarrizes the acceleration responses from the sweep frequency tests. When the current changed from $0 \mathrm{~A}$ to $2 \mathrm{~A}$, the peak acceleration appears at $28.81 \mathrm{~Hz}$ and $49.92 \mathrm{~Hz}$, respectively

As known, to obtain a better control performance in displacement when the excitation is periodic loading, it would be desirable to stiffen the isolator. On the other hand, for better mitigation of the acceleration, stiffening the isolator only leads to excessive acceleration. Ideal situation for acceleration reduction is to tune the natural frequency of the isolator to be far less than the excitation frequency. However, soft isolator, e.g. very low natural frequency, leads to excessive displacement transmitted to the isolated object. Therefore, trade-off must be considered during the design of the control law if the criteria are to have both displacement and acceleration controlled in certain range. Reviewing the experimental results, if only considering isolated displacement, the minimum displacement profile occurs only when the applied current is $0 \mathrm{~A}$ or $2 \mathrm{~A}$, shown in Figure 5. There is a threshold frequency where below this frequency one should apply 2.0A and above this frequency one should apply $0 \mathrm{~A}$, in order to obtain optimal displacement reduction.

(a)

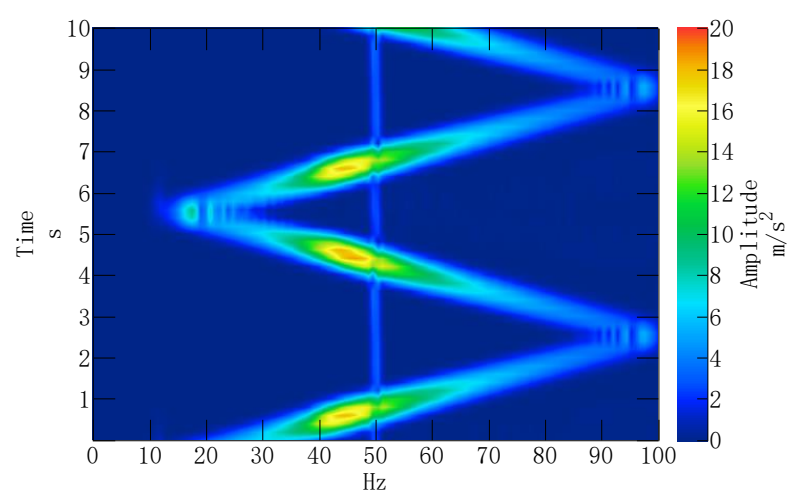

(c)

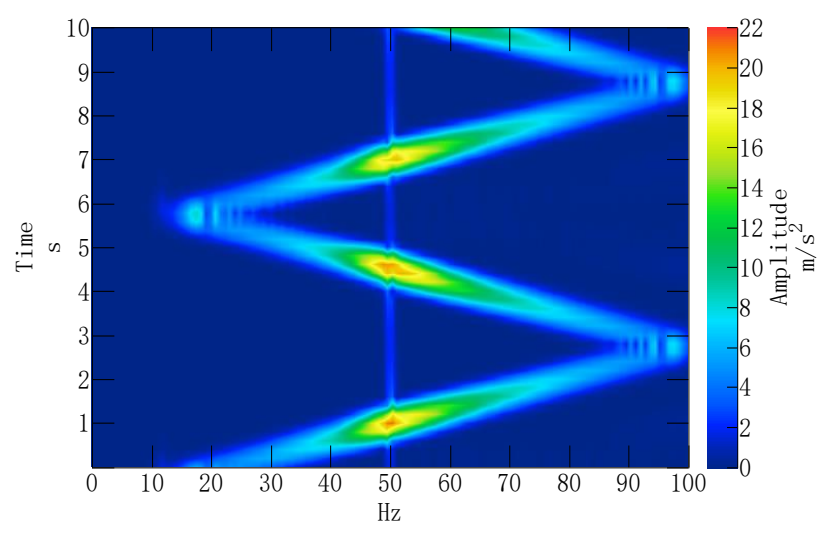

(e)

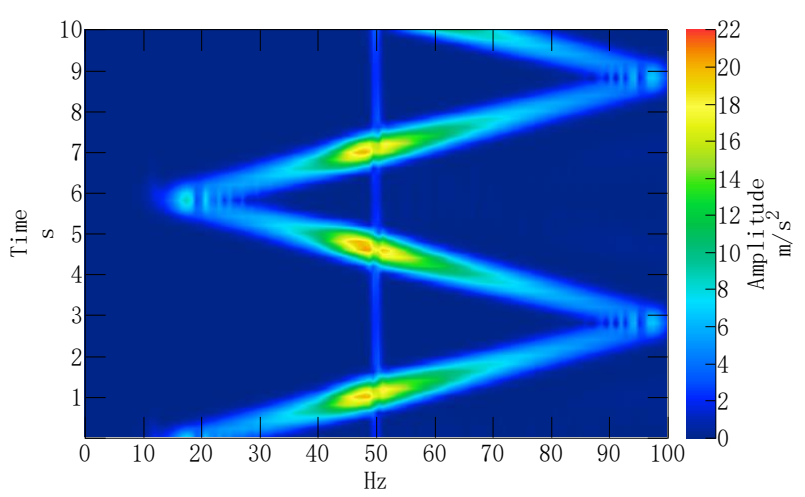

(d)

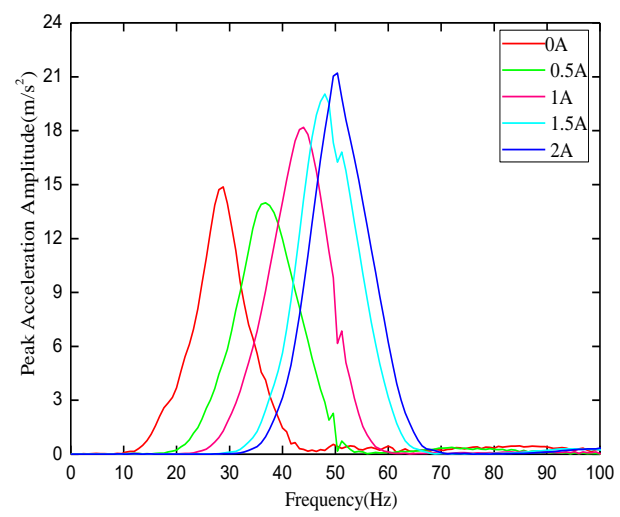

(f)

Figure 6: The test result at sweep-frequency excitations: (a) the acceleration amplitude spectrum under current 0A, (b) the acceleration amplitude spectrum under current $0.5 \mathrm{~A}$, (c) the acceleration amplitude spectrum under current $1 \mathrm{~A}$, (d) the acceleration amplitude spectrum under current $1.5 \mathrm{~A}$, (e) the acceleration amplitude spectrum under current $2 \mathrm{~A}$, (f) the spice of acceleration amplitude spectrum under different current.

\section{Mathematical modeling}

MRE is a kind of viscoelastic material that can be described by Kelvin model [34]. MRE vibration isolation system can be simplified as a single degree of freedom system. The mathematical model of the SAPII vibration isolation system is shown in figure 7.

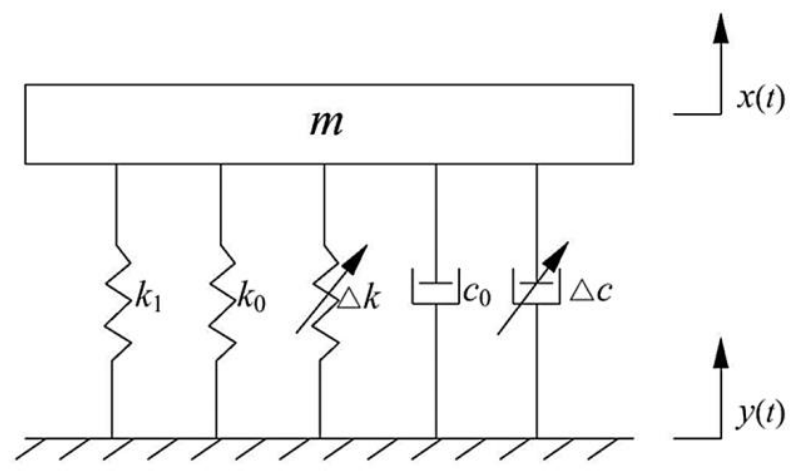


Figure 7: The mathematical model of the SAPII vibration isolation system

The governing equation of this system could be derived as

$$
m \ddot{x}+\left(c_{0}+\Delta c\right)(\dot{x}-\dot{y})+\left(k_{0}+k_{1}+\Delta k\right)(x-y)=0
$$

where $k_{1}$ is the stiffness of the spring, specified as $8.04 \mathrm{Nmm}^{-1} \cdot k_{0}$ and $c_{0}$ are the stiffness and damping of the MRE isolator under zero magnetic field, respectively. $\Delta k$ and $\Delta c$ are the stiffness and the damping increment caused by magnetic field separately. $m$ is the mass of payload, including the mass block, the platform and the support block. $x(\mathrm{t})$ is the displacement response for displacement excitation $y(\mathrm{t})$.

The key parameters about stiffness and damping can be expressed as

$$
\begin{aligned}
& k_{M}=k_{0}+\Delta k \\
& k=k_{0}+k_{1}+\Delta k \\
& c=c_{0}+\Delta c
\end{aligned}
$$

where, $k_{\mathrm{M}}$ represents the stiffness of MRE, and $k, c$ represent equivalent stiffness and equivalent damping, respectively.

The equation (1) can be simplified as

$$
m \ddot{x}+c(\dot{x}-\dot{y})+k(x-y)=0
$$

The force provided by MRE can be represented as

$$
F_{M R E}=k_{M} \cdot R D+c \cdot R V
$$

where $\mathrm{RD}$ and $\mathrm{RV}$ represent relative displacement and relative velocity, separately.

The control force induced by magnetic field can be written as

$$
F_{C}=\Delta k \cdot R D+\Delta c \cdot R V
$$

Therefore, the governing equation of this system could also be derived as

$$
m \ddot{x}+c_{0}(\dot{x}-\dot{y})+\left(k_{0}+k_{1}\right)(x-y)+F_{C}=0
$$

By applying the Laplace transform on both sides of the equation (5), we can get the transfer function of the SAPII vibration isolation system written as follows

$$
G(S)=\frac{c s+k}{m s^{2}+c s+k}
$$

The displacement transmissibility of the SAPII vibration isolation system can be represented as

$$
T=\left|\frac{X}{Y}\right|=\sqrt{\frac{1+(2 \zeta \lambda)^{2}}{\left(1-\lambda^{2}\right)^{2}+(2 \zeta \lambda)^{2}}}
$$

where $\lambda=\frac{f}{f_{n}}, f_{n}=\frac{1}{2 \pi} \sqrt{\frac{k}{m}}, \zeta=\frac{c}{4 \pi m f_{n}} . f$ is the excitation frequency, and $f_{\mathrm{n}}$ is the natural frequency of the isolation system.

For a single degree of freedom system with damping, the resonance occurs in

$$
f=f_{d}=\sqrt{\left(1-2 \zeta^{2}\right)} f_{n}=\frac{1}{2 \pi} \sqrt{\frac{2 m k-c^{2}}{2 m^{2}}}
$$

where $f_{\mathrm{d}}$ is the damped resonance frequency of the SAPII vibration isolation system.

At this time, the displacement transmissibility of the SAPII vibration isolation system reached its peak $T_{\mathrm{m}}$ calculated by

$$
T=T_{m}=\sqrt{\frac{k^{2}+c^{2}\left(2 \pi f_{d}\right)^{2}}{k^{2}+m^{2}\left(2 \pi f_{d}\right)^{4}-2 m k\left(2 \pi f_{d}\right)^{2}+c^{2}\left(2 \pi f_{d}\right)^{2}}}
$$


From equation (11) and equation (12), the equivalent stiffness and the equivalent damping can be represented as follows

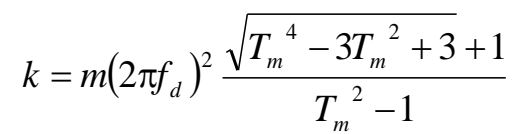

$$
\begin{aligned}
& c=\sqrt{2} m^{2}\left(2 \pi f_{d}\right)^{2} \frac{\sqrt{T_{m}{ }^{4}-3 T_{m}{ }^{2}+3}-T_{m}{ }^{2}+2}{\sqrt{T_{m}{ }^{2}-1}}
\end{aligned}
$$

$f_{\mathrm{d}}$ and $T_{\mathrm{m}}$ can be obtained from the transmissibility curves under different currents shown in figure 5. Here, $m$ is specified as $0.7 \mathrm{~kg}$. The parameters of the SAPII vibration isolation system, such as equivalent stiffness, equivalent damping, nature frequency, resonance frequency, damping ratio listed in table 1 . Under applied current $0-2 \mathrm{~A}$, the relative change of the equivalent stiffness is $218.69 \%$ and the relative change of the equivalent damping is $332.82 \%$.

Table 1.The parameters of the SAPII vibration isolation system

\begin{tabular}{cccccc}
\hline$I$ & $0 \mathrm{~A}$ & $0.5 \mathrm{~A}$ & $1 \mathrm{~A}$ & $1.5 \mathrm{~A}$ & $2 \mathrm{~A}$ \\
\hline$T_{\mathrm{m}}$ & 5.348 & 4.238 & 3.600 & 2.933 & 2.421 \\
$f_{\mathrm{d}}(\mathrm{Hz})$ & 30.0 & 34.3 & 39.4 & 48.2 & 51.0 \\
$f_{\mathrm{n}}(\mathrm{Hz})$ & 30.198 & 34.822 & 40.268 & 49.921 & 53.908 \\
$\zeta$ & 0.096 & 0.122 & 0.146 & 0.184 & 0.232 \\
$k\left(\mathrm{Nmm}^{-1}\right)$ & 25.20 & 33.51 & 44.81 & 68.87 & 80.31 \\
$c\left(\mathrm{~N} \cdot \mathrm{sm}^{-1}\right)$ & 25.393 & 37.454 & 51.720 & 80.829 & 109.907 \\
$\Delta k\left(\mathrm{Nmm}^{-1}\right)$ & 0 & 8.31 & 19.61 & 43.28 & 55.11 \\
$\Delta c\left(\mathrm{~N} \cdot \mathrm{sm}^{-1}\right)$ & 0 & 12.061 & 26.327 & 55.436 & 84.514 \\
$k_{M}\left(\mathrm{Nmm}^{-1}\right)$ & 17.16 & 25.47 & 36.77 & 60.83 & 72.27 \\
\hline
\end{tabular}

\section{Control strategies for the SAPII vibration isolation system}

\subsection{Sinusoid Excitation}

The demand to isolate periodic excitations from mechanical systems is often requested. Using MRE variable stiffness mechanism, Liao et al [19] has proposed a simple ON-OFF control law with aim to reduce the transmitted displacement, as following:

$$
\begin{cases}k=k_{\max } \text { and } c=c_{\max } & \text { if } \omega \leq \sqrt{2} \omega_{0} \\ k=k_{\min } \text { and } c=c_{\min } & \text { if } \omega>\sqrt{2} \omega_{0}\end{cases}
$$

Where, $k_{\max }$ and $k_{\min }$ are the maximum and minimum stiffness of the MRE isolation system, respectively. $c_{\max }$ and $c_{\min }$ are the maximum and minimum damping of the MRE isolation system, respectively. $\omega$ is the excitation frequency and $\omega_{0}$ is the natural frequency of the MRE isolation system. The proposed control law utilizes the natural frequency of the MRE system as control feedback. However, such reference frequency is not fixed while switching between ON and OFF states which lays challenge to implement such control law.

As observed from the experiment, the minimum peak of displacement always appears in 0A state or 2A state as shown in figure 5. A simple ON-OFF control law is therefore proposed as follows:

$$
I= \begin{cases}2 A & f \leq f_{0} \\ 0 A & f>f_{0}\end{cases}
$$


Where, $f_{0}$ is the threshold frequency. The ON-OFF threshold frequency is the abscissa of intersection point of displacement transmissibility functions under $0 \mathrm{~A}$ state and $2 \mathrm{~A}$ state.

For a similar system with changeable spring stiffness, considering spring stiffness as a variable, the equivalent stiffness under $0 \mathrm{~A}$ and $2 \mathrm{~A}$ can be calculated by equation 17 and equation 18 :

$$
\begin{aligned}
& k_{O A}=17.16+k_{1} \\
& k_{2 A}=72.27+k_{1}
\end{aligned}
$$

From equation (10), (17)-(18), the ON-OFF threshold can be expressed as a function of $k_{1}$ and $m$ (the payload mass) as shown in figure 8 . When payload and spring change, the ON-OFF threshold can be adjusted according the mass and the spring stiffness. Here, the ON-OFF threshold $f_{0}$ corresponding to the mass of $0.7 \mathrm{~kg}$ and the spring stiffness of 8.04 $\mathrm{Nmm}^{-1}$ in the system is $37.536 \mathrm{~Hz}$.



Figure 8. ON-OFF threshold surface

\subsection{Random Excitation}

Random excitation is another encounter in vibration isolation applications. In this part, two control algorithms, i.e. ON-OFF control and fuzzy control, are designed to take full advantage of the proposed system.

\subsubsection{ON-OFF control law}

The ON-OFF control for the random excitation is the integration of damping ON-OFF control $[35,36]$ and stiffness ON-OFF control [37]. The ON-OFF control law can be expressed as

$$
\begin{cases}k=k_{\max } \text { and } c=c_{\max } & \text { if } R D \cdot R V \geq 0 \\ k=k_{\min } \text { and } c=c_{\text {min }} & \text { if } R D \cdot R V<0\end{cases}
$$

Where $k_{\max }$ and $k_{\min }$ are the maximum and minimum stiffness of the SAPII, respectively. $c_{\max }$ and $c_{\min }$ are the maximum and minimum damping of the SAPII, respectively. $R D$ and $R V$ represent payload's displacement and velocity with respect to base, respectively. $R D \cdot R V \geq 0$ indicate the load is moving away from the equilibrium position. In this time, the selection of large damping coefficient is helpful to restrain this trend. When the load is approaching to the equilibrium position respect to $R D \cdot R V<0$, the small damping switches the SAPII into soft state resulting the payload to return to the 
equilibrium position easily. The control mechanism is illustrated in figure 9[19]. In the SPII system, stiffness and damping raise with the increase of applied current. Therefore, the ON-OFF control law can be further summarized as

$$
I= \begin{cases}2 A & R D \cdot R V \geq 0 \\ 0 A & R D \cdot R V<0\end{cases}
$$

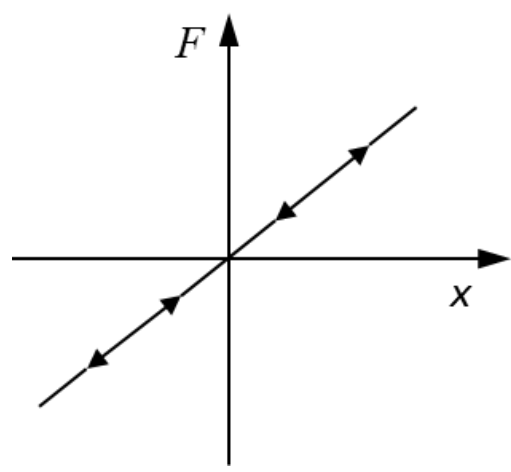

(a)

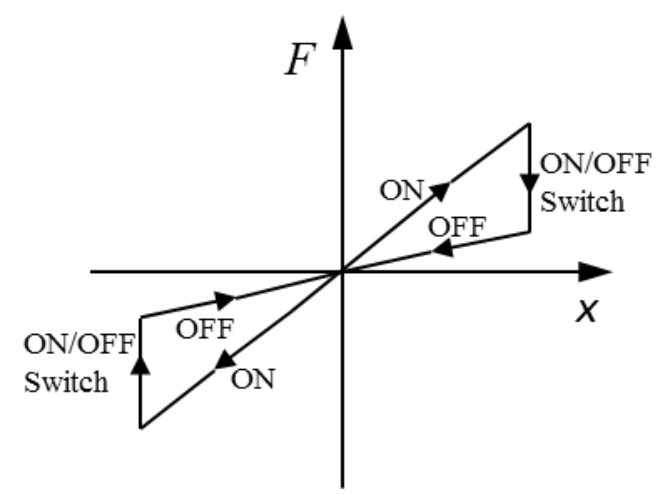

(b)

Figure 9. Stiffness ON-OFF control schematic: (a) without control and (b) ON-OFF control

\subsubsection{Fuzzy logic controller}

To achieve robust performance with system uncertainties, a fuzzy logic controller is designed shown in figure 10. The fuzzy logic controller is nested in a closed-loop system including fuzzifier, inference mechanism, defuzzifier and fuzzy rules. $I$ is the applied current, which represents the input of the controlled system and the output of the fuzzy logic controller. $x, r$ is the payload's displacement and reference displacement, respectively. Here, the expected reference displacement is set to zero. $e$ represent the deviation between payload's displacement and reference displacement. $c$ is the change rate of the payload's displacement. $e, c$ is the input of the fuzzy logic controller, which can be expressed as equation (21) and equation (22), respectively. $k_{e}, k_{c}$ and $k_{i}$ are the quantification factors of $e, c$ and $I$.

$$
\begin{gathered}
e(t)=r(t)-x(t)=-x(t) \\
c(t)=\frac{d}{d t} e(t)=-\dot{x}(t)
\end{gathered}
$$

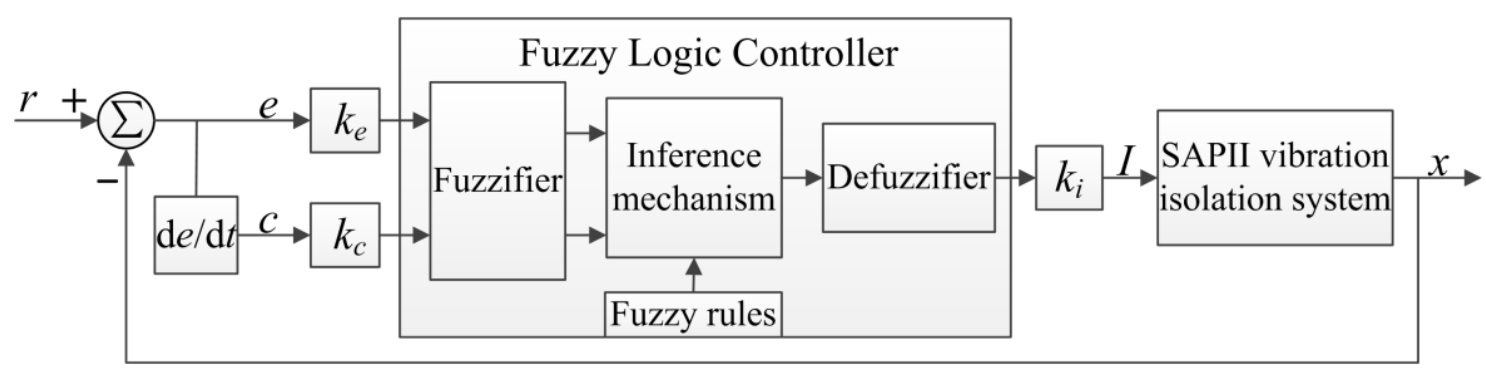

Figure 10. The structure of the fuzzy logic controller

Considering the characteristic of the controller, the input linguistic variables are assigned by using seven fuzzy subsets, which are denoted by negative big (NB), negative medium (NM), negative small (NS), zero (ZO), positive small (PS), positive medium (PM), and positive big (PB). The output linguistic variables are assigned by using four fuzzy subsets, which are denoted by zero (ZO), positive small (PS), positive medium (PM), and positive big (PB). The discourse domain of the input is set as $[-2,2]$ while the discourse domain of the output is set as $[0,2]$. The membership functions of the input and the output are illustrated in figure 11 and figure 12. The membership functions of the input belong to 
gaussmf-type to enhance the smoothness of fuzzefier. The membership functions of the output are triangle-type to improve the sensitivity of defuzzifier. The fuzzy inference mechanism is based on max-min inference based on Mamdani 's method. The center-of-gravity method is introduced to defuzzifier.

In this paper, the fuzzy logic controller includes 49 pieces of fuzzy rules listed in table 2 . Two exemplar rules are listed as follows

1. If $e(t)$ is $\mathrm{PB}$ and $c(t)$ is $\mathrm{PB}$ then $I$ is $\mathrm{PB}$

2. If $e(t)$ is NM and $c(t)$ is PS then $I$ is PS

Rule 1 indicates that the fuzzy logic controller should output a larger current to restrain the rapid growth when the payload has a large positive displacement and a large increase trend.

Rule 2 means that the fuzzy logic controller should output a smaller current to pull the payload back to equilibrium position when the load has a medium negative displacement and a slight decrease trend.

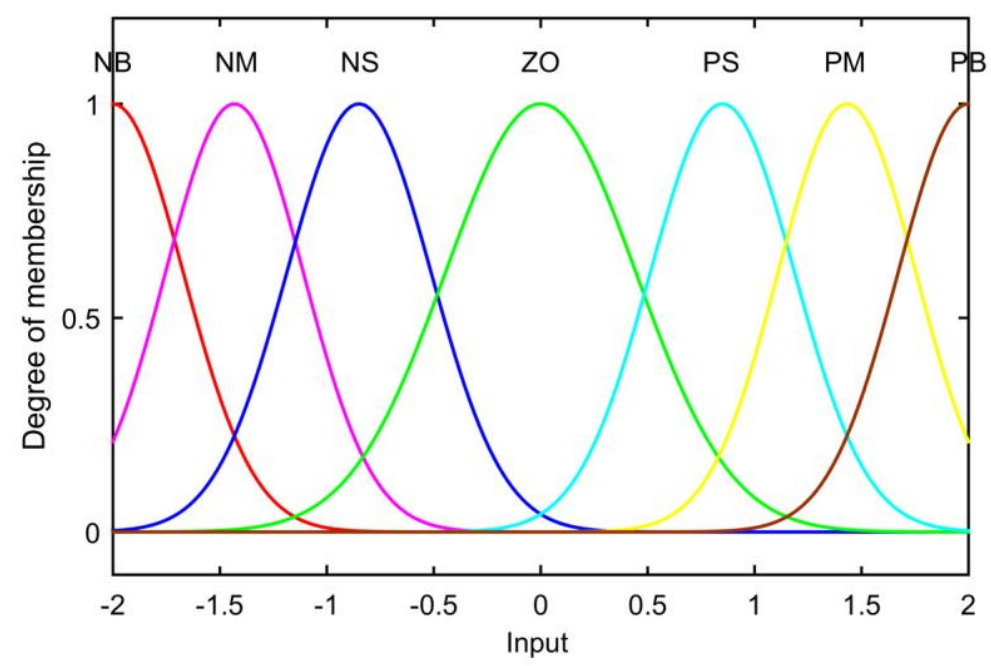

Figure 11. The membership function of the inputs

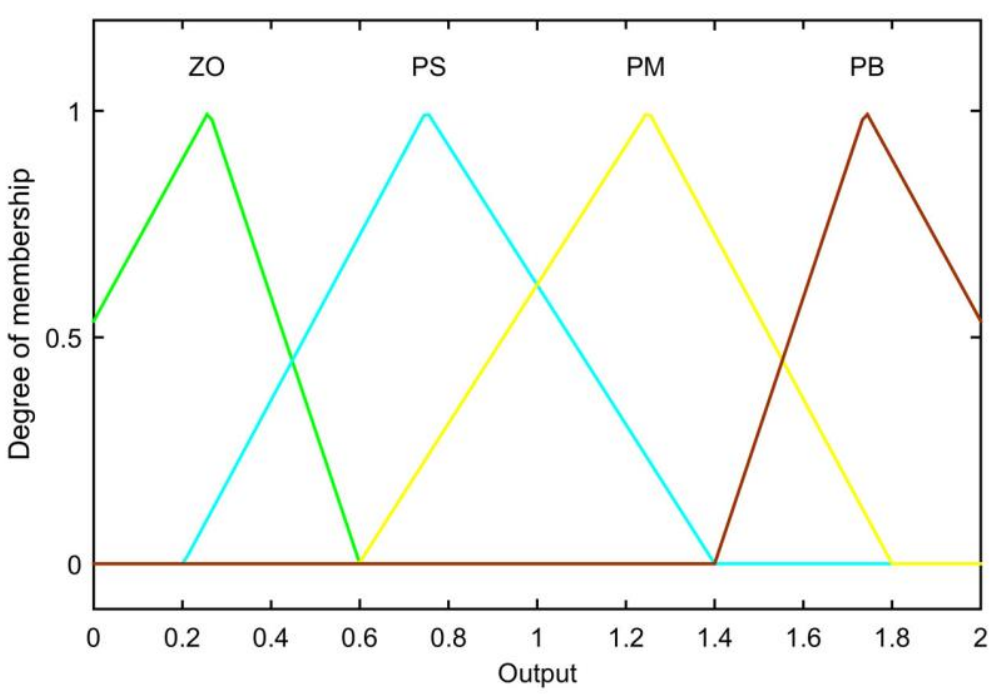

Figure 12. The membership function of the outputs 
Table 2.The fuzzy rules of the fuzzy logic controller

\begin{tabular}{ccccccccc}
\hline \multirow{2}{*}{\multicolumn{1}{c}{$I$}} & \multicolumn{8}{c}{$c(t)$} \\
\cline { 2 - 8 }$e(t)$ & NB & PB & PB & PB & PB & PM & PS & ZO \\
& NM & PB & PB & PM & PM & PS & ZO & PS \\
& NS & PB & PM & PM & PS & ZO & PS & PM \\
& ZO & PB & PM & PS & ZO & PS & PM & PB \\
& PS & PM & PS & ZO & PS & PM & PM & PB \\
& PM & PS & ZO & PS & PM & PM & PB & PB \\
& PB & ZO & PS & PM & PB & PB & PB & PB \\
\hline
\end{tabular}

\section{Experiment and simulation}

\subsection{Experiment results for sweep-frequency sinusoid excitation}

The experimental setup to investigate the ON-OFF control performance of SPII vibration isolation system under sweep-frequency excitation is shown in figure 13. A laser displacement sensor and an acceleration sensor are upgraded in this experiment setup compared with dynamic response characteristic experiment setup. The DSP (Model TMS320F28335, Texas Instruments, USA) is used as the processor of the vibration isolation system. The other apparatuses are the same as shown in figure 4. The excitation signal, produced by lower displacement sensor, is transformed by 12-bite A/D converter in DSP. The frequency of excitation can be calculated by FFT algorithm built in DSP controller.



Figure 13. The experimental schematic of the SAPII vibration isolation system with an ON-OFF controller The experiment results of the control performance under $25-80 \mathrm{~Hz}$ sweep-frequency sinusoid excitation are presented in figure 14. Both passive and ON-OFF control can achieve good isolation performance in displacement and acceleration responses over wide frequency range excitation compared with excitation. However, the passive control demonstates its 
incapacity in reducing displacement and acceletration in resonant frequency, around $30 \mathrm{~Hz}$. The ON-OFF control, on the other hand, proves its effectiveness over entire frequency band. The comparison of the experiment results for sweep-frequency excitation under passive and ON-OFF condition is summarized in table 3 . As can be observed, both the maximum values and RMS values of displacement and acceleration responses have been greatly reduced for ON-OFF control compared with passive system. The reduction on displacement is about $50 \%$ while the reduction on acceleration response is about $40 \%$.

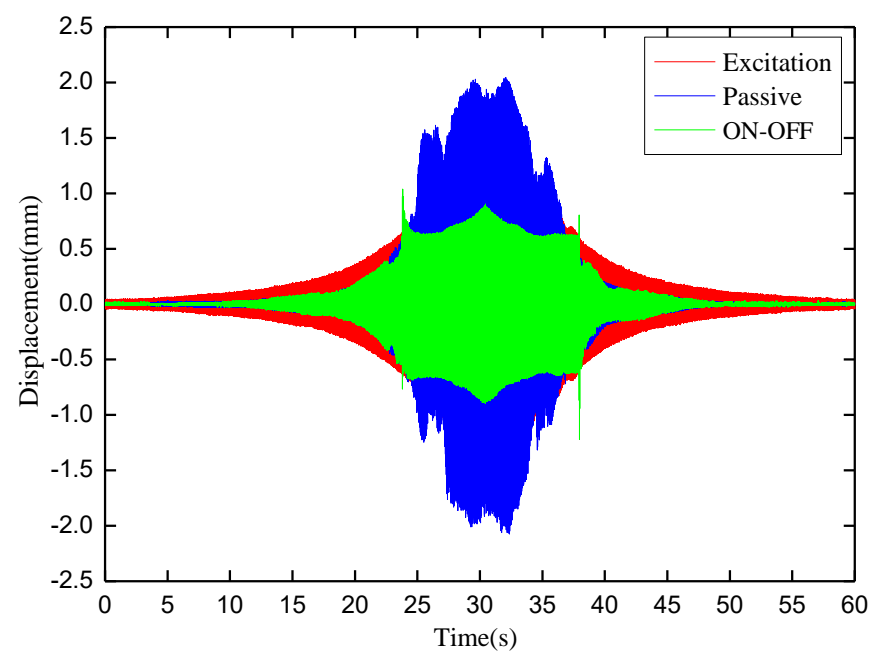

(a)

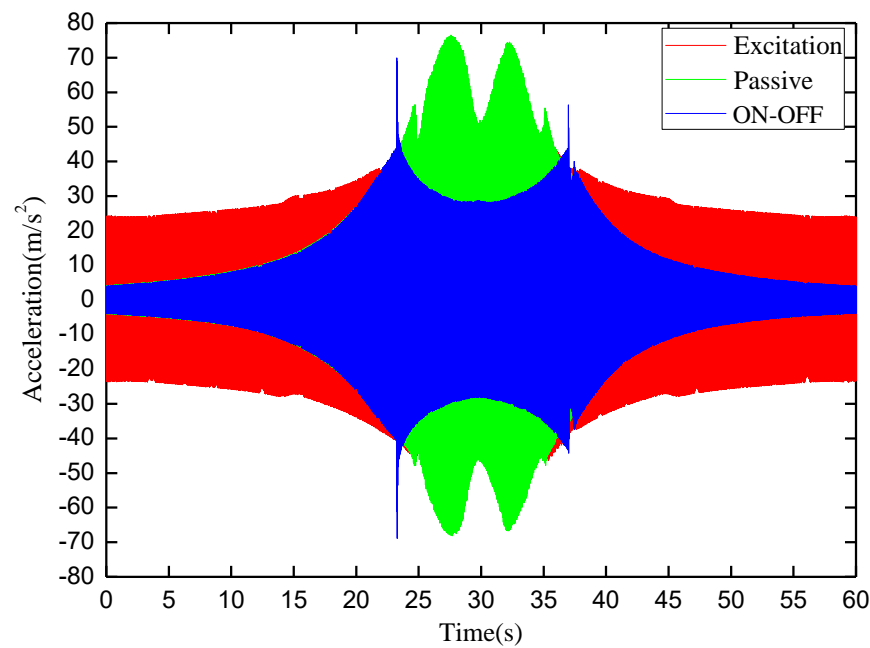

(b)

Figure 14. The experiment results under $25-80 \mathrm{~Hz}$ sweep-frequency excitation: (a) the displacement comparison among excitation, passive and ON-OFF control, (b) the acceleration responses comparison among excitation, passive and ON-OFF control

Table 3.Comparison of the experiment results for sweep-frequency excitation under passive and ON-OFF control

\begin{tabular}{lccccc}
\hline & \multicolumn{2}{c}{ Maximum values } & & \multicolumn{2}{c}{ RMS values } \\
\cline { 2 - 3 } \cline { 5 - 6 } & Displacement $(\mathrm{mm})$ & Acceleration $\left(\mathrm{m} / \mathrm{s}^{2}\right)$ & & Displacement $(\mathrm{mm})$ & Acceleration $\left(\mathrm{m} / \mathrm{s}^{2}\right)$ \\
\hline Passive & 1.990 & 76.519 & & 0.496 & 20.723 \\
ON-OFF & 0.902 & 45.505 & & 0.251 & 14.445 \\
\hline
\end{tabular}




\subsection{Numerical analysis on control performance of $\mathrm{ON}-\mathrm{OFF}$ and fuzzy logic controllers under random excitation}

To verify the effectiveness of proposed control algorithms for random excitation, Simulink model is created as showed in figures 15 and 16. The random excitation source is made of Band-Limited White Noise module and Analog Filter. The frequency range of the random excitation is chosen around the resonant frequency, i.e. $20-35 \mathrm{~Hz}$, due to it's vulnerability at its resonant frequency range. Figure 17 illustrates the time history of the random excitation and its frequency spectrum. The system properties of the SPAII are as followings: payload $m=0.7 \mathrm{~kg}$ and the spring stiffness $k=8.04 \mathrm{Nmm}^{-1}$. The maximum allowable current is $I_{\max }=2.0 \mathrm{~A}$ for both passive and fuzzy logic control. In the fuzzy logic control system, the amplification factors are $k_{e}=1800, k_{c}=8.5$ and $k_{i}=1$, respectively. Other system parameters are selected same as in Table 1 .

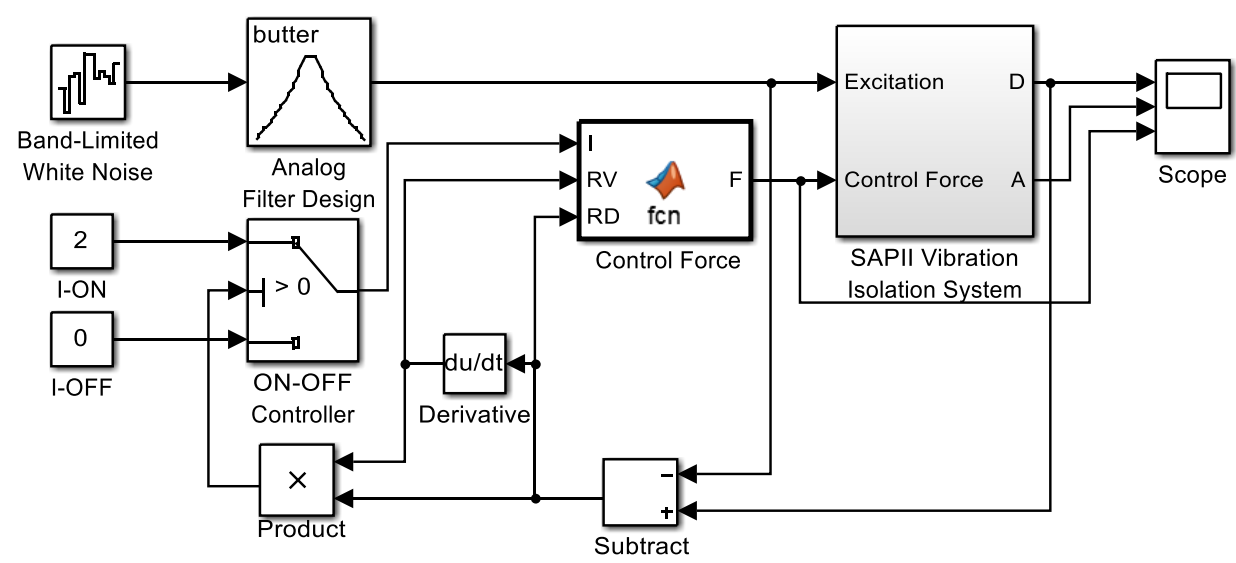

Figure 15. The Simulink model for ON-OFF control under random excitation

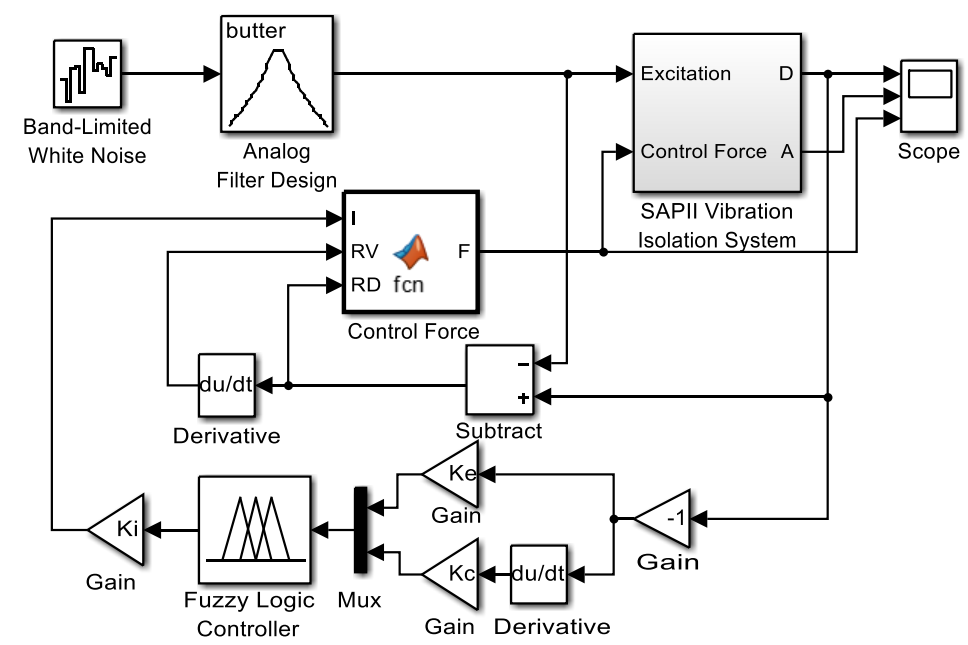

Figure 16. The Simulink model for fuzzy logic control under random excitation 


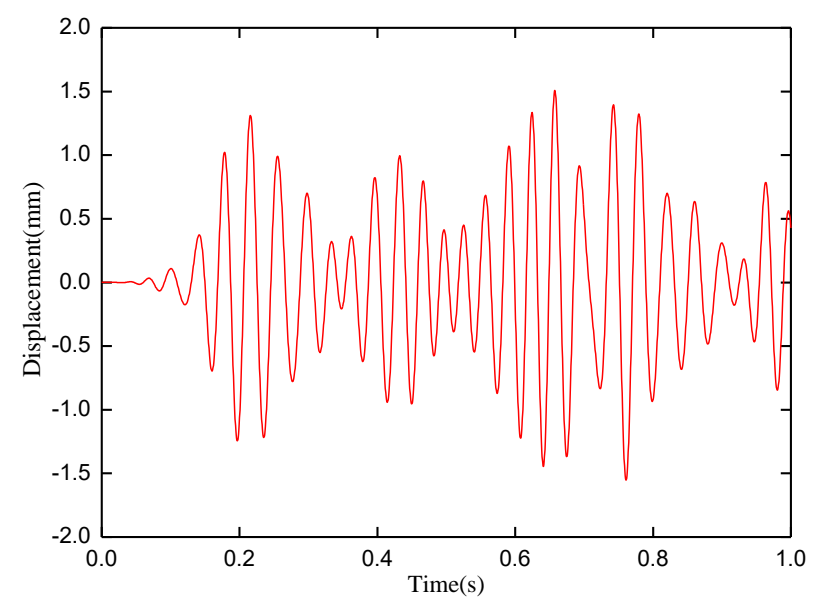

(a)

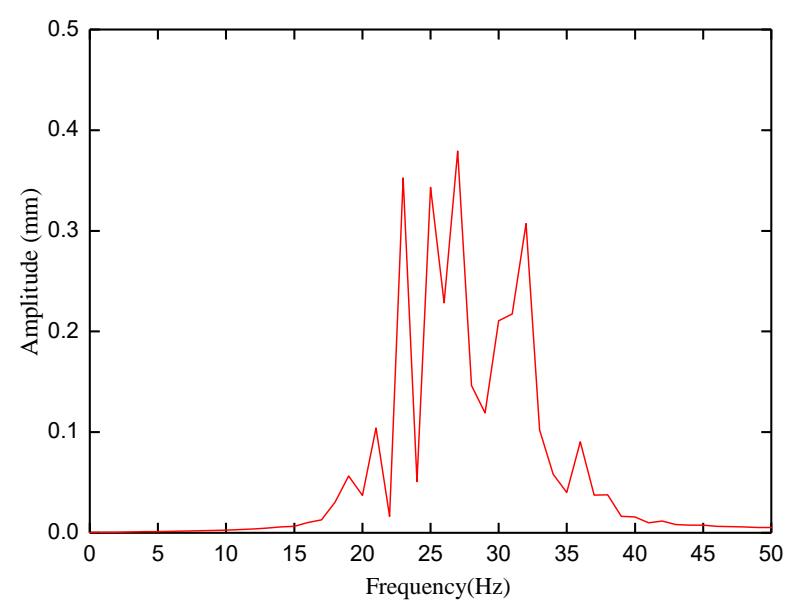

(b)

Figure 17. Random excitation: (a) time history; (b) frequency spectrum

Figure 18 shows the performances of the SPAII isolation system with passive, ON-OFF control and fuzzy logic control. For both displacement and acceleration responses, the controlled systems, i.e. with ON-OFF controland fuzzy logic control, have much better performances than uncontrolled one, i.e. passive system. ON-OFF control achieves similar displacement reduction compared with fuzzy logic system. However, fuzzy logic controller surpasses ON-OFF controller in acceleration isolation. Table 4 gives the statistical sumarry of the control performance for three systems.

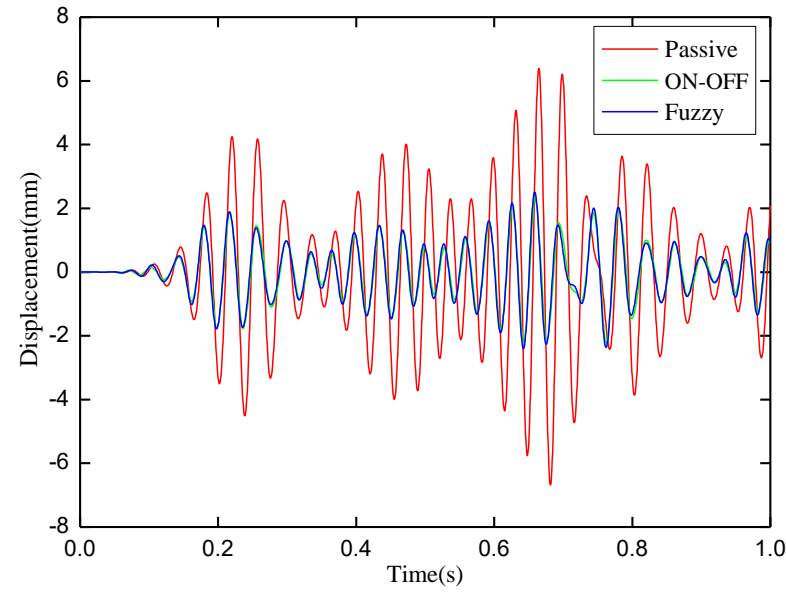

(a)

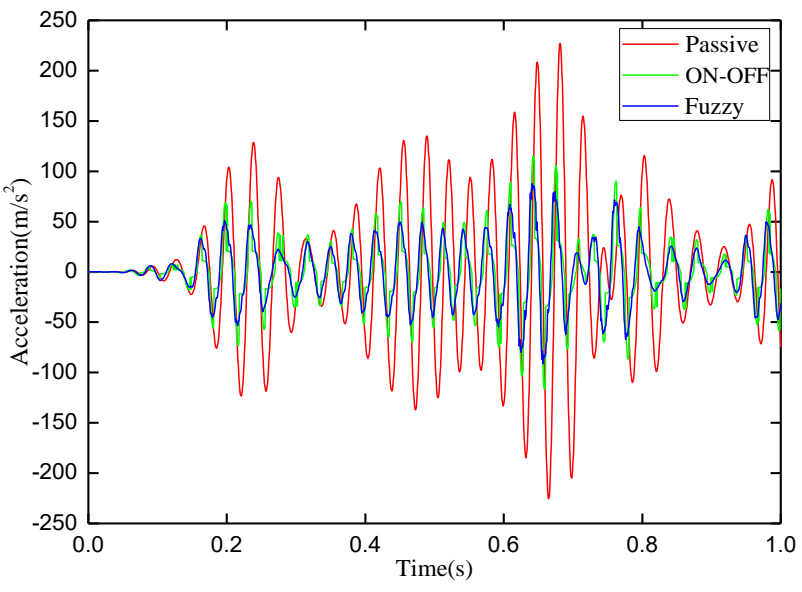

(b)

Figure 18. Performance comparison between passive, ON-OFF and fuzzy logic control: (a) displacement responses; (b) acceleration responses;

Table 4.Comparison of the simulation results for random excitation under passive, ON-OFF and fuzzy condition

\begin{tabular}{|c|c|c|c|c|}
\hline & \multicolumn{2}{|c|}{ Maximum values } & \multicolumn{2}{|c|}{ RMS values } \\
\hline & Displacement $(\mathrm{mm})$ & $\operatorname{Acceleration}\left(\mathrm{m} / \mathrm{s}^{2}\right)$ & Displacement $(\mathrm{mm})$ & $\operatorname{Acceleration}\left(\mathrm{m} / \mathrm{s}^{2}\right)$ \\
\hline Passive & 6.679 & 227.033 & 2.168 & 66.788 \\
\hline ON-OFF & 2.356 & 116.448 & 0.859 & 30.265 \\
\hline Fuzzy & 2.507 & 91.340 & 0.905 & 29.770 \\
\hline
\end{tabular}


To further review the control performance, two parameters are presented in figure 19, i.e. control force and control current. Apparently, fuzzy logic control possesses smoother transition in the applied currents while maintaining optimal control forces. In the view of energy consumption and electronic design of the control system, fuzzy logic control would be better choice for implementation.

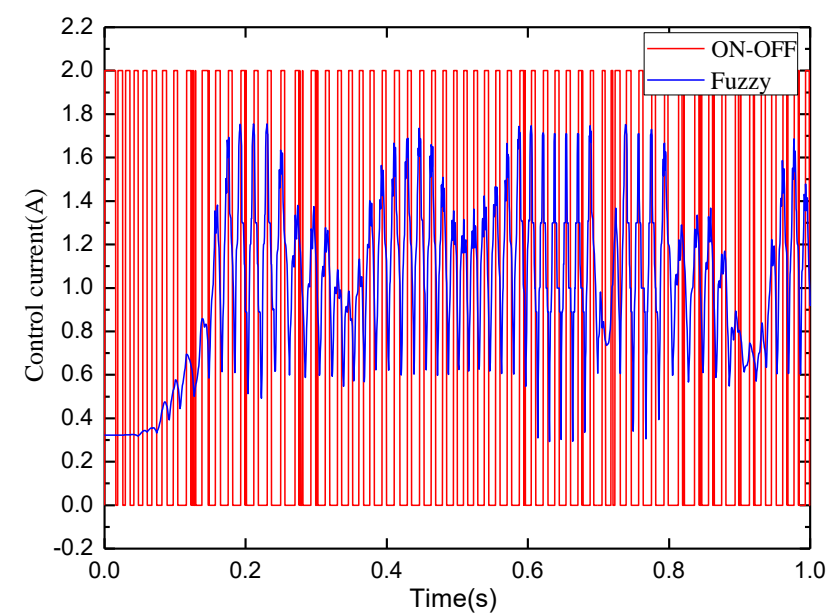

(a)

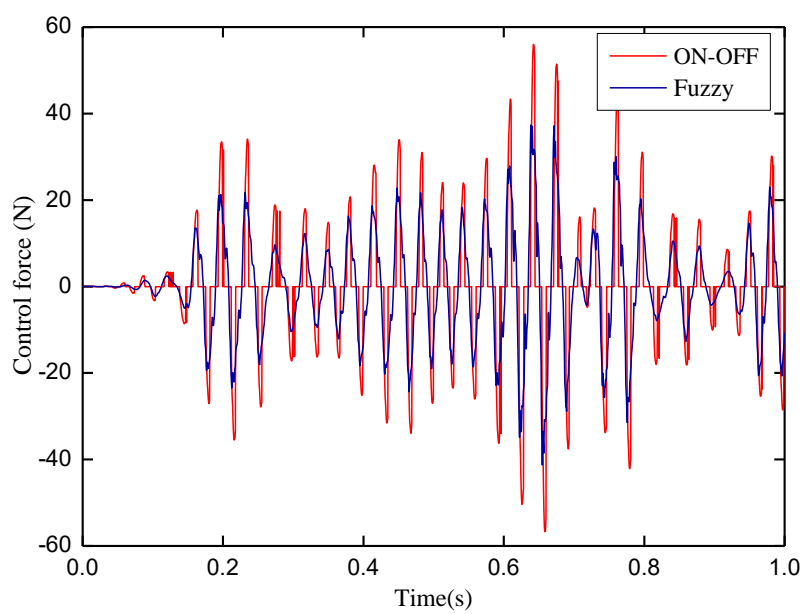

(b)

Figure 19. Comparison between ON-OFF control and fuzzy logic control: (a) control currents; (b)control forces;

\section{Conclusions}

A semi-active/passive integrated isolator (SAPII) is designed to achieve effective vibration isolation in a wide frequency range by using MRE and spring elements. To obtain the vibration isolation performance of the SAPII, dynamic response characteristic test is performed. The sweep-frequency excitation test illustrates that the resonant frequencies of the SPAII can be tuned from $30.0 \mathrm{~Hz}$ (without applied current) to $51.0 \mathrm{~Hz}$ (with applied current of 2A). The dynamic model of the SAPII vibration isolation system is then established built on Kelvin model. The formulas to identify key parameters, such as equivalent stiffness and equivalent damping, are derived from the dynamic model and displacement transmissibility curve. Under applied currents from $0 \mathrm{~A}$ to $2 \mathrm{~A}$, the relative increase of the equivalent stiffness of SPAII is $218.69 \%$. If considering the stiffness increase of MRE only, it's stiffness change of MRE up to 321.15\%, from initial stiffness of 17.16 $\mathrm{Nmm}^{-1}$ to $72.27 \mathrm{Nmm}^{-1}$ when the applied current is 2.0A. An ON-OFF control law based on the minimum displacement transmissibility is proposed for the control of the sinusoid excitation. Experimental results shows the ON-OFF control law implemented can eliminate resonance peak evidently to achieve effective vibration isolation for a wide-frequency sinusoid excitation. Two control laws are proposed for vibration isolation of random excitation, i.e. ON-OFF control and fuzzy logic control. With superior performance on acceleration reduction as well as energy comsumtion, the fuzzy logic control is recommended real applications.

\section{References}

[1]. Ellison J and Ahmadi G 2001 Passive vibration control of air borne equipment using a circular steel ring Journal of Sound and vibration 246 1-28 
[2]. Cunningham D and Davis P 1993 A multiaxis passive isolation system for a magnetic bearing reaction wheel Advances in the Astronautical Sciences 9580426

[3]. Eugene I and Rivin 2003 Passive Vibration Isolation ASME Press 57 B31-B32

[4]. Housner G W, Bergman L A, Caughey T K, Chassiakos A G and Claus R O 1997 Structural control: Past, present, and future Journal of Engineering Mechanics 123 897-971

[5]. Yu Y, Nagi G N and Rao V D 2001 A literature review of automotive vehicle engine mounting systems Mechanism and Machine Theory 36 123-142

[6]. Kim Y K, Koo J H, Kim K S and Kim S H 2011 Suppressing harmonic vibrations of a miniature cryogenic cooler using an adaptive tunable vibration absorber based on magneto-rheological elastomers Review of Scientific Instruments 82035103

[7]. Carlson J D and Jolly M R 2000 MR fluid foam and elastomer devices Mechatronics 10 555-69

[8]. Ginder J M, Nichols M E, Elie L D and Clark S M 2000 Controllable-stiffness components based on magnetorheological elastomers Proc. SPIE 3985 418-25

[9]. Li Y, Li J, Li W and Samali B 2013 Development and characterization of a magnetorheological elastomer based adaptive seismic isolator Smart Mater. Struct. 22035005

[10]. Jolly M R, Carlson J D and Munoz B C 1996 A model of the behaviour of magnetorheological materials Smart Mater. Struct. 5 607-614

[11]. Behrooz M, Wang X and Gordaninejad F 2014 Performance of a new magnetorheological elastomer isolation system Smart Mater. Struct. 23045014

[12]. Deng H and Gong X 2007 Adaptive tuned vibration absorber based on magnetorheological elastomer J. Intell. Mater. Syst. Struct. 18 1205-10

[13]. Zhang X and Li W 2009 Adaptive tuned dynamic vibration absorbers working with MR elastomers Smart Struct. Syst. 5 517-29

[14]. Xu Z, Gong X, Liao G and Chen X 2010 An active-damping-compensated magnetorheological elastomer adaptive tuned vibration absorber J. Intell. Mater. Syst. Struct. 21 1039-47

[15]. Liao G, Gong X, Kang C J and Xuan S 2011 The design of an active-adaptive tuned vibration absorber based on magnetorheological elastomer and its vibration attenuation performance Smart Mater. Struct. 20 145-151

[16]. Sun S, Chen Y, Yang J, Tian T, Deng H, Li W, Du H and Alici G 2014 The development of an adaptive tuned magnetorheological elastomer absorber working in squeeze mode Smart Mater. Struct. 23075009

[17]. Kim Y K, Bae H I, Koo J H, Kim K S and Kim S 2012 Real time control of a tunable vibration absorber based on magnetorheological elastomer for suppressing tonal vibrations Rev. Sci. Instrum. 83046108

[18]. Li W, Zhang X and Du H 2012 Development and simulation evaluation of a magnetorheological elastomer isolator for seat vibration control J. Intell. Mater. Syst. Struct. 23 1041-48

[19]. Liao G, Gong X, Xuan S H, Kang C J and Zong L 2012 Development of a real-time tunable stiffness and damping vibration isolator based on magnetorheological elastomer J. Intell. Mater. Syst.Struct.23 25-33

[20]. Li J, Li Y, Li W and Samali B 2013 Development of adaptive seismic isolators for ultimate seismic protection of civil structures Proceedings of SPIE - The International Society for Optical Engineering 8692

[21]. Li Y, Li J, Tian T, Li W 2013 A highly adjustable magnetorheological elastomer base isolator for applications of real-time adaptive control Smart Mater. Struct. 22095020

[22]. Lerner A A and Cunefare K A 2008 Performance of MRE-based vibration absorber J. Intell. Mater. Syst. Struct. $19551-63$

[23]. Ni Z, Gong X, Li J and Chen L 2009 Study on a dynamic stiffness-tuning absorber with squeeze-strain 
enhanced magnetorheological elastomer J. Intell. Mater. Syst. Struct. 23 1195-202

[24]. Kavlicoglu B, Wallis B, Sahin H and Liu Y 2011 Magnetorheological elastomer mount for shock and vibration isolation Proceedings of SPIE - The International Society for Optical Engineering 79770Y-7

[25]. Kallio M, Lindroos T, Aalto S, Jarvinen E, Kama T and Meinander T 2007 Dynamic compression testing of a tunable spring element consisting of a magnetorheological elastomer Smart Mater. Struct. 16 506-14

[26]. Du H, Li W, Zhang N 2011 Semi-active variable stiffness vibration control of vehicle seat suspension using an MR-elastomer isolator Smart Mater. Struct. 20105003

[27]. Jeong UC, Yoon J H, Yang I, Jeong J E, Kim J S, Chung K H and Oh J E 2013 Magnetorheological elastomer with stiffness-variable characteristics based on induced current applied to differential mount of vehicles Smart Mater. Struct. 22115007

[28]. Fu J, Li P, Wang Y, Liao G and Yu M 2016 Model-free fuzzy control of a magnetorheological elastomer vibration isolation system: analysis and experimental evaluation Smart Mater. Struct. 25035030

[29]. Opie S and Yim W 2011 Design and control of a real-time variable modulus vibration isolator J. Intel. Mat. Syst. Str. 22 113-25

[30]. Li G, Huang X and Wang J 2013 Preparation of magnetorheological elastomer and mechanical properties research Materials review 27 36-39

[31]. Li Y, Li J, Li W and Du H 2014 A state-of-the-art review on magnetorheological elastomer devices Smart Mater. Struct. 23123001

[32]. Chen L, Gong X 2008 Damping of magnetorheological elastomers Journal of Central South University 21 $271-274$

[33]. Li J and Gong X 2008 Dynamic damping property of magnetorheological elastomer Journal of Central South University 15 261-265

[34]. Chang K, Lai M, Soong T T, Oh S T D S and Yeh Y C 1993 Seismic behavior and design guidelines for steel frame structures with added viscoelastic dampers Technical Report Nceer 93-0009

[35]. Liu Y, Matsuhisa H and Utsuno H 2008 Semi-active vibration isolation system with variable stiffness and damping control Journal of Sound and Vibration 313 16-28

[36]. Jansen L M and Dyke S J 2000 Semiactive control strategies for MR dampers-Comparative study Journal of Engineering Mechanics 126 795-803

[37]. Nagarajaiah S, Asce M and Sonmez E 2007 Structures with semiactive variable stiffness single/multiple tuned mass dampers Journal of Structural Engineering 133 67-77 